\title{
Space Weather Storm Responses at Mars: Lessons from A Weakly Magnetized Terrestrial Planet
}

\author{
J. G. Luhmann ${ }^{1}$, C. F. Dong ${ }^{2}$, Y. J. Ma ${ }^{2}$, S. M. Curry ${ }^{1}$, Yan Li ${ }^{1}$, \\ C. O. Lee $^{1}$, T. Hara ${ }^{1}$, R. Lillis ${ }^{1}$, J. Halekas ${ }^{4}$, J. E. Connerney ${ }^{5}$,
} J. Espley ${ }^{5}$, D. A. Brain ${ }^{6}$, Y. Dong ${ }^{6}$, B. M. Jakosky ${ }^{6}$, E. Thiemann ${ }^{6}$,

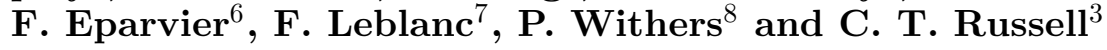

${ }^{1}$ Space Sciences Laboratory, University of California, Berkeley, CA, USA, email: jgluhman@ssl. berkeley.edu

${ }^{2}$ Princeton University, Princeton, NJ, USA

${ }^{3}$ Institute of Geophysics and Planetary Physics, UCLA, Los Angeles, CA, USA

${ }^{4}$ University of Iowa, Iowa City, IA, USA

${ }^{5}$ NASA Goddard Space Flight Center, Greenbelt, MD, USA

${ }^{6}$ LASP, University of Colorado, Boulder, CO, USA

${ }^{7}$ LATMOS/IPSL, UPMC University Paris, Paris, France

${ }^{8}$ Boston University, Boston, MA, USA

\begin{abstract}
Much can be learned from terrestrial planets that appear to have had the potential to be habitable, but failed to realize that potential. Mars shows evidence of a once hospitable surface environment. The reasons for its current state, and in particular its thin atmosphere and dry surface, are of great interest for what they can tell us about habitable zone planet outcomes. A main goal of the MAVEN mission is to observe Mars' atmosphere responses to solar and space weather influences, and in particular atmosphere escape related to space weather 'storms' caused by interplanetary coronal mass ejections (ICMEs). Numerical experiments with a data-validated MHD model suggest how the effects of an observed moderately strong ICME compare to what happens during a more extreme event. The results suggest the kinds of solar and space weather conditions that can have evolutionary importance at a planet like Mars.
\end{abstract}

Keywords. Mars, Magnetosphere, Magnetic Storm

\section{Introduction}

Exploration of the terrestrial planets over the last $\sim 5$ decades has shown how diverse the outcome of their evolutionary paths can be. The many influences include both internal processes and interactions with their external environments, including the Sun and solar wind, over time. Of the other planets in or near our star's currently habitable zone, Mars has the particular distinction of having been observed since telescopes came into existence. It moreover appears to have been a possible abode of life early in its history, and is a natural destination for human exploration. As a consequence a host of space missions, including orbiters, landers and rovers, have been sent to carry out robotic investigations of the state of Mars' surface and atmosphere, with the Viking landers in the 70s having searched for evidence of extant life on or near the surface. Today we know a great deal about the solid planet, whose radius is roughly half that of Earth, from extensive topographical, mineralogical and gravitational mapping by orbiters. We know that Mars has a highly multipolar crustal magnetic field that, while strong compared to Earth's field in some locations, falls of relatively rapidly in strength with altitude. We also know from the landed platforms and remote sensing that the atmosphere is thin 
( $\sim 10$ mb surface pressure compared to Earth's 1 bar), and composed mainly of carbon dioxide. Both the atmosphere and surface are relatively dry as well, though there is a strong seasonal variation in both carbon dioxide and water content as the polar ice caps periodically sublime over a Mars year when exposed to greater solar illumination and warming. Huge dust storms on this dry planet are also observed to produce significant heating effects in the upper atmosphere and ionosphere (e.g., Jakosky and Phillips (2001).

Interest has steadily grown in how Mars came to its present state, with surface features and mineralogy both suggesting it once had (or has) at least occasional flowing water episodes with enough volume to create the many channels observed from the ground and space- and possibly once a northern ocean. The isotopic evidence moreover supports the interpretation that a significant part of the original (post-impact-period) atmosphere of Mars, including its water, either has been lost through mineralogical alteration and sequestration of carbon, oxygen and other constituents, or has escaped. Much work continues on evaluating the still present reservoirs and the processes of surface and subsurface uptake. But comparably intensive work on the atmosphere escape side only started in earnest with the Mars Express mission (e.g. Lundin et al. (2013) and references therein), stimulated in part by the discovery by Mars Global Surveyor of small-scale remanent magnetic fields- presumably left from an early era of planetary dynamo operation (Acuna et al. (1999)). A popular, though still widely-debated idea holds that planetary magnetospheres can 'shield' a planetary atmosphere from processes that lead to massive escape on evolutionary timescales. Mars Express (MEX) mainly measured the escaping ionized atmospheric species, which constitute only part of the overall escaping population. Theory and modelling expectations, bolstered by related observations at Venus, have long suggested a (currently) more important contribution at Mars from escaping suprathermal or 'hot' oxygen atoms produced by dissociative recombination in its mainly $\mathrm{O} 2+$ ionosphere, with a possible contribution from sputtering by precipitating planetary ions (e.g. Lillis et al. (2015)). But the historical extrapolation of integrated loss that includes these and other possible escape processes has been compromised by the lack of a more complete complement of related measurements toward evaluating them.

The MAVEN (Mars Atmosphere and Volatile EvolutioN) mission has been in Mars orbit for over a Mars year with a broad instrument complement designed to investigate atmosphere escape processes and consequences (Jakosky et al. (2016)). In addition to solar wind and planetary plasma spectrometers and a magnetometer, it includes a thermal ion and neutral mass spectrometer, a UV spectrograph and EUV photometer designed to detect the rarefied exospheric gases and solar activity indicators respectively, and solar energetic particle telescopes (Jakosky et al. (2015a)). Over the course of is prime mission period (late 2014 through 2015), it has observed the variability of the upper atmosphere and ionosphere, and measured atmospheric ion escape rates under a range of conditions. Of particular interest here is that MAVEN finds the average global escape rates of both ions and neutrals, derived with the aid of global extrapolations of data-validated models, are too low by several orders of magnitude to explain the loss of the one or more bar atmosphere inferred to have been present $\sim 3.5$ Gyr ago. This age is an often adopted benchmark in time because the evidence from cratering records and surface magnetic field measurements indicate that the remanent field has been present since that time, with the implication that any global dynamo was terminated or decayed away by then.

The reasons why loss to space by currently active processes has not been abandoned as a still feasible explanation for the apparent loss of the atmosphere are twofold. One strong argument is the well-established isotopic evidence, for which there is little alternative explanation (although see Guedel et al. (2010), for a discussion of escape processes prior to the 3.5 Gyr mark). The other involves the growing inferences from observations of 
Sun-like stars of the early Sun's properties, and in particular its probable higher level of magnetic activity. Flare responses in the form of upper atmosphere heating, with implied increased thermal escape, have been observed on MAVEN (Thiemann et al. (2015)), though the involved time intervals are brief. But MEX and MAVEN observations have both indicated order-of-magnitude increases in escaping atmospheric ion fluxes when an interplanetary coronal mass ejection (ICME), with its enhanced solar wind plasma and field parameters lasting a day or more, passes Mars (e.g. Edberg et al. (2010); Jakosky et al. (2015b)). A potentially answerable question relevant to Mars, and more general planetary atmosphere evolution, concerns how large escape rates due to current processes can become, and whether these can explain the evident loss of a potentially habitable surface environment.

In this paper we focus on the specific process of atmospheric ion escape, neglecting the related sputtering and independent photochemical losses to obtain a lower limit for this cometary ion tail-like erosion. Global ion escape rates for both quiet times and times of enhanced solar wind parameters have been determined from MAVEN observations with the aid of MHD simulations of the solar wind interaction (Jakosky et al. (2015b); Dong et al. (2015)). We first revisit the case when enhanced solar wind conditions occurred when an ICME passed Mars in March 2015. As previously described, inferred global planetary ion outflow enhancements occur at different stages during that $\sim 1.5$ day-long event, which involves an initial shock/sheath phase with compressed solar wind plasma and field, followed by an ejecta phase dominated by the strong field of the coronal erupted material or driver. The model gives a sense of the complex topology of the Mars magnetosphere reconfiguration and ionosphere energization that leads to the increased ion escape. We then ask how much larger such event-related ion escape rates might become, at least by present day standards, by using radially extrapolated solar wind parameters from a particularly extreme ICME event observed at 1 AU by the STEREO-A spacecraft in July 2012. By modelling the Mars atmospheric ion escape response to this example we obtain a better idea of how proposed stronger solar activity conditions on the young Sun might have regularly affected the early Mars atmosphere, at least through this single process of ion escape.

\section{Description of the disturbed external conditions and model results}

The results of the simulations shown here are described in more detail in a paper submitted to the MAVEN special issue of the Journal of Geophysical Research (Luhmann et al. (2016)). Basic CO2 atmosphere photochemistry in the models produces an ionosphere composed of $\mathrm{O} 2+, \mathrm{O}+$ and $\mathrm{CO} 2+$ major ion species affected by the solar wind interaction in a self-consistent manner. In the single fluid MHD approximation used, details of the ion escape relating to the heavy atmospheric ion gyroradii (e.g. Dong et al. (2015)) are not included. However, it has been found that in spite of this simplification the single fluid treatment can describe spacecraft observations of the magnetic fields, thermal ions, and solar wind around Mars extremely well (Ma et al. (2015)). We therefore assume that even above the exobase, physical process that act like collisions determine much of the basic solar wind interaction character, including the bulk planetary plasma energization by the interaction. Within the MHD framework there are several mechanisms that energize the atmospheric ions, including solar wind convection electric fields mapped along open field lines into the ionosphere in a manner similar to what happens in Earth's high latitude open field regions, magnetic field and thermal pressure gradient forces, and magnetic tension forces. Details not explicitly included are the energy deposition and sputtering effects of those accelerated planetary ions whose trajectories carry 


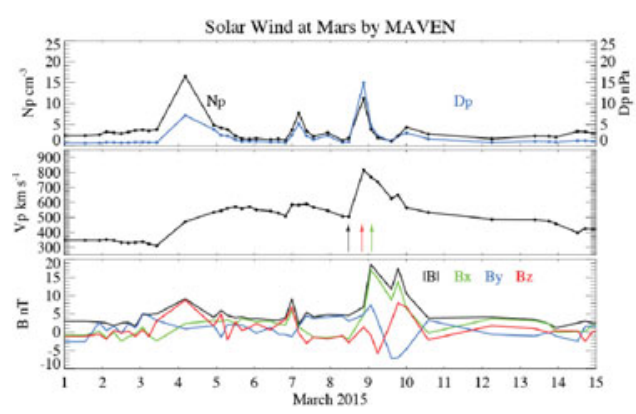

(a)

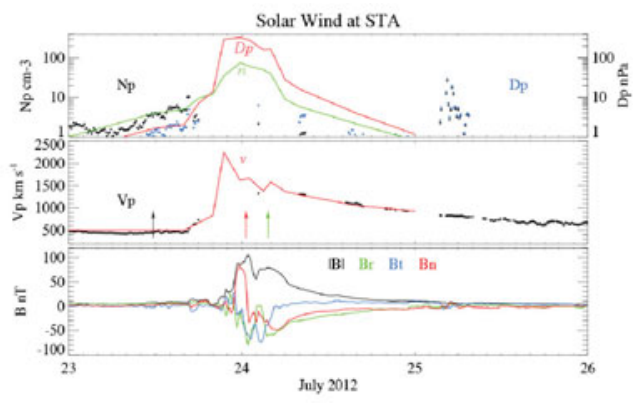

(b)

Figure 1. In-situ time series showing the interplanetary conditions (solar wind plasma density and velocity and magnetic field) observed by MAVEN at Mars in March 2015, including the passage of an ICME on March 8-10 (left panel), and a much larger event observed at $1 \mathrm{AU}$ on STEREO-A in July 2012 (right panel). The latter can be used to simulate the effects of a realistic, more extreme event at Mars (see text)

them back into the atmosphere (see Curry et al. (2015)), related ion outflow reductions, wave-particle interactions, and fluid instabilities at the solar wind-ionosphere boundary. However, because of the apparent data-model agreement, these effects can be considered second-order in the current context.

As discussed above, we are interested in the more extreme interplanetary conditions experienced today, which occur when major interplanetary coronal mass ejection disturbances pass. The in-situ solar wind conditions for the March 2015 and July 2012 ICMEs at Mars and at STEREO-A respectively are shown in Figures 1a and 1b. Even accounting for the $0.5 \mathrm{AU}$ heliocentric distance difference, it can be seen that the notable ICME observed at Mars on March 9-10, 2015 pales in comparison to the STEREO-A event. This provides important perspective because of the likelihood of more frequent strong activity on the early Sun (e.g. Ribas et al. (2005)).

The times indicated by arrows in Figures 1a,b were used to define the external parameters during each event for BATS-R-US solar wind interaction runs, assuming the same, nominal Mars atmosphere and solar EUV fluxes appropriate for each period. These represent the pre-ICME conditions in the solar wind, the piled up solar wind or ICME sheath phase when the incident dynamic pressure is largest, and the driver or ejecta phase when the magnetic field dominates the plasma pressure. As STEREO-A is located at 1 AU, in the July 2012 event case, the magnetic fields and densities were scaled to Mars heliocentric distance. The basic parameters used in the simulations for the pre-event and peak incident pressures for both events are summarized in Table 1 together with the related ion escape rates.

The enhancement of the planetary ion fluxes around Mars obtained with the solar wind interaction simulation for the more extreme July 2012 external conditions is illustrated in Figure 2 which shows (log) flux contours in a noon-midnight meridian cross section (where $\mathrm{x}$ is the Mars-Sun axis and $\mathrm{z}$ points north from the Mars orbital plane). These represent the Mars response to the external conditions in Figure 1b before the ICME hit (black arrow) and during the peak of the ICME's incident dynamic pressure (red arrow). The superposed black lines are projections of the models' 3D magnetic field lines traced from a circle of points located at $150 \mathrm{~km}$ altitude in the plane of the contour plot. These show how the planetary ion flux structure is influenced by the combination of the interplanetary magnetic field and Mars' crustal magnetic field. At the passage of the ICME, the magnetic fields of Mars undergo enhanced reconnection with the strong 
Table 1.

\begin{tabular}{|c|c|c|c|c|}
\hline Case & Nsw $\left(\mathrm{cm}^{-3}\right)$ & Vsw $(\mathrm{km} / \mathrm{s})$ & B (nT) & Ion Escape $(\# / \mathrm{s}) \mid$ \\
\hline PreMar2015 ICME & 1.8 & 505 & 4.8 & $1.5 \cdot 10^{24}$ \\
\hline PmaxMar2015 & 11.0 & 817 & 7.7 & $1.1 \cdot 10^{25}$ \\
\hline Pre STA ICME & 0.8 & 480 & 2.6 & $3.9 \cdot 10^{24}$ \\
\hline Pmax STA ICME & 24.8 & 1700 & 54.9 & $1.4 \cdot 10^{27}$ \\
\hline
\end{tabular}

Notes:values for March 2015 are from Curry et al. (2015), and for the STA event from Luhmann et al. (2016)

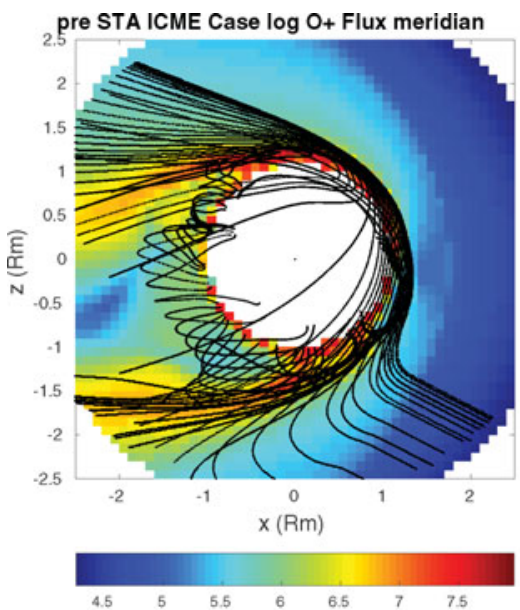

(a)

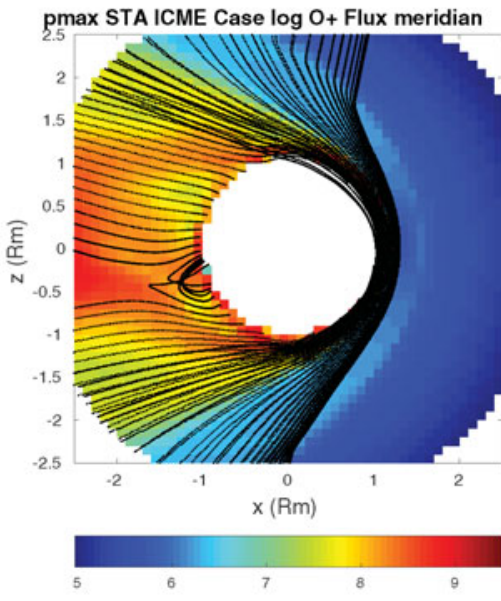

(b)

Figure 2. In-situ time series showing the interplanetary conditions (solar wind plasma density and velocity and magnetic field) observed by MAVEN at Mars in March 2015, including the passage of an ICME on March 8-10 (left panel), and a much larger event observed at $1 \mathrm{AU}$ on STEREO-A in July 2012 (right panel). The latter can be used to simulate the effects of a realistic, more extreme event at Mars (see text)

external fields. As a result, the solar wind interaction looks more Venus-like on the dayside because the ionosphere induced fields are too weak to exclude deep magnetosheath field penetration and pile-up. However, away from the subsolar region reconnection produces 'open' Martian magnetosphere fields that nearly fill the solar wind wake. The consequence is an ionosphere that is much more widely exposed to solar wind convection electric fields, and thus widely subject to outward thermal pressure gradient forces. Magnetic reconnection also enhances the ion outflows.

As discussed in Luhmann et al. (2016), the several order of magnitude escape rate enhancement seen in the extreme case can have a significant impact on Mars atmosphere escape on evolutionary time scales. However, this depends on whether similar interplanetary conditions were sufficiently long-lived or extreme events were more common.

\section{Implications from Sun-as-a-Star Research for Mars and Exoplanet Research}

Several papers presented at this conference have addressed important issues relevant to using present-day observations of Mars ion escape to infer how much of its atmosphere could have been lost as a result of this ion escape process acting over the past $\sim 3-4$ billion 
years. Guedel et al. (2010) points out the recent results from Tu et al. (2015) suggesting order-of-magnitude uncertainties in the solar ionizing fluxes in the early period prior to 3.5 Gyr ago. As the solar EUV flux evolution figures prominently in historical escape estimates, this represents an important uncertainty that must be taken into account in backward extrapolations of present escape processes including not only ion escape, but also neutral escape mechanisms (photochemical production and escape of hot atoms, in particular) not included here. The ion loss models discussed here all use a nominal present day EUV flux at Mars.

Another important uncertainty is the extent to which the planets experienced enhanced solar wind parameters simply from a stronger early solar wind. The implication of Airapetian and Usmanov's (2016) evolving solar wind models is that between $~ 700$ Myr and a few Gyr of age, the Sun produced a solar wind with nominal speeds of up to $\sim 800-1400 \mathrm{~km} / \mathrm{s}$, velocities that today are associated with major ICMEs. Moreover, the much higher mass flux of this early solar wind (by up to several orders of magnitude) would have produced regularly high plasma dynamic pressures on the same order as those during the ICMEs described here. The magnetic fields at the solar surface, assumed to be $\sim 3-4 \mathrm{x}$ higher than the present Sun's based on stellar analogs, combined with the higher younger Sun rotation rates (periods 5-10 days in their models) would have increased the interplanetary magnetic field by at least as much. Moreover, these already higher fields and dynamic pressures would be regularly enhanced by the formation of stream interaction regions, which would have corotated by at the higher solar rotation rates. With these relatively intense ambient conditions prevailing over a billion years, loss rates on the order of the ICME loss rates described here should have routinely occurred even without coronal eruptions. The fact that the Airapetian and Usmanov early solar wind model mass fluxes compare favorably with those inferred by Wood (2006) from astrosphere observations lends credence to this possible early ion escape scenario.

At the same time, estimates of increased flaring activity on the young Sun from the stellar analogs suggest those were also part of its history (e.g. Maehara et al. (2012)). Moreover, the flare energies may have been larger by orders of magnitude. The implications for the related early interplanetary medium are likely to remain unclear. As suggested by other speakers at this conference (e.g. Osten et al. (2017)), it has been difficult to establish the existence of the stellar counterparts of coronal mass ejections, although work is ongoing. Some efforts to imagine how a superflare would affect the planets have been made but they are necessarily speculative. Our broad challenge is to find ways to constrain scenarios for star-planet interactions over their lifetimes. Solar system observations like those from the MAVEN mission at Mars can help provide some 'ground truth' for reality checks, as described here.

\section{Acknowledgments}

This work has been supported by NASA through contracts to the Laboratory for Atmospheric and Space Physics at the University of Colorado, Boulder, in support of the MAVEN Mission. The authors are grateful to all who made the MAVEN mission possible, and also to the developers of the BATS-R-US MHD model at the University of Michigan, Ann Arbor and the NASA High End Computing Program.

\section{References}

Acuna, M. H. et al., Global distribution of crustal magnetization discovered by the Mars Global Surveyor 1999, Science, 284, 790-793 
Airapetian, V. S. \& Usmanov, A. V. Reconstructing the solar wind from its early history to current epoch 2016, ApJ, 817, L24-L30

Curry, S.M., et al., Response of Mars O+ pickup ions to the 8 March 2015 ICME: Inferences from MAVEN data-based models 2015, Geophys. Res. Lett., 42, 9095-9102

Dong, C. F., et al., 2015, Multifluid MHD study of the solar wind interaction with Mars' upper atmosphere during the 2015 March 8 th ICME event 2015, Geophys. Res. Lett., 42, 91039112

Edberg, N. J. T. , Nilsson, H., A. O. Williams, M. Lester, S. E. Milan, S. W. H.. Cowley, M. Fraenz, S. Barabash, \& Y. Futaana, Pumping out the atmosphere of Mars through solar wind pressure pulses 2010, Geophys. Res. Lett., 37,

Guedel et al., this volume

Jakosky, B. \& Phillips, R., Mars' volatile and climate history 2001, Nature, 412, 237-244

Jakosky, B. M. et al., 2015a, The Mars atmosphere and volatile evolution (MAVEN) mission 2015a, Space Sci. Rev., 195,

Jakosky, B. M. et al., 2015b, MAVEN observations of the response of Mars to an interplanetary coronal mass ejection 2015b, Science, 350,

\&Jakosky, B. M., J. M. Grebowsky, J. G. Luhmann, \& D. A. Brain, The MAVEN mission to Mars at the end of one Mars year of science observations 2016, J. Geophys. Res., in press

Lillis, R. et al., Characterizing atmospheric escape from Mars today and through time 2015, Space Sci. Rev., 195, 357-422

Luhmann, J. G., C. F. Dong, Y. J. Ma, S. M. Curry, S. Xu, C. O. Lee, T. Hara, J. Halekas, \& Yan Li, J. R. Gruesbeck, J. Espley, D. A. Brain, \& C. T. Russell, 2016, Martian magnetic storms 2016, submitted to J. Geophys. Res.

\&Lundin, R., S. Barabash, M. Homstrom, H. Nillson, Y. Futaana, R. Ramstad, M. Yamauchi, E. Dubinin, \& M. Fraenz, Solar cycle effects on the ion escape from Mars 2013, Geophys. Res. Lett., 40, 6028-6032

Ma, Y. J., et al., MHD model results of solar wind interaction with Mars and comparison with MAVEN plasma observations 2015, Geophys. Res. Lett., 42, 9113-9120

\&Maehara, , H., T. Shibayama, S. Notsu, Y. Notsu, T. Nagao, S. Kusaba, S. Honda, D. Nogami, \& K. Shibata, Superflares on solar-type stars 2012, Nature, 485, 478-481

Osten et al., this volume

Ribas, L., E. F. Guinan, M. Guedel, \& M. Audard, Evolution of the Solar Activity over Time and Effects on Planetary Atmospheres. I. High-Energy Irradiances $(1-1700+)$ 2005, ApJ, 622, 680-694

Terada, N., Y. N. Kulikov, H. Lammer, H. Lichtenegger, T. Tanaka, H. Shinagawa, \& T. Zhang, Atmosphere and water loss from early Mars under extreme solar wind and extreme ultraviolet conditions 2009, Astrobiology, 9, 55-70

Thiemann, E. M., et al., Neutral density response to solar flares at Mars 2015, Geophys. Res. Lett., 42, 8986-8992

\&Tu, L., C. P. Johnstone, M. Guedel, \& H. Lammer, The Extreme Ultraviolet and X-Ray Sun in Time: High-Energy Evolutionary Tracks of a Solar-Like Star 2015, Astronomy and Astrophysics, 577

Wood, B. E., The Solar Wind and the Sun in the Past 2006, Space Sci. Rev., 126, 3-14 